\title{
Functional comparison of the nematode Hox gene lin-39 in C. elegans and $P$. pacificus reveals evolutionary conservation of protein function despite divergence of primary sequences
}

\author{
Kaj Grandien ${ }^{1}$ and Ralf J. Sommer ${ }^{2}$ \\ Max-Planck Institut für Entwicklungsbiologie, Abteilung Evolutionsbiologie, D-72076 Tübingen, Germany
}

\begin{abstract}
Hox transcription factors have been implicated in playing a central role in the evolution of animal morphology. Many studies indicate the evolutionary importance of regulatory changes in Hox genes, but little is known about the role of functional changes in Hox proteins. In the nematodes Pristionchus pacificus and Caenorhabditis elegans, developmental processes can be compared at the cellular, genetic, and molecular levels and differences in gene function can be identified. The Hox gene lin-39 is involved in the regulation of nematode vulva development. Comparison of known lin-39 mutations in P. pacificus and C. elegans revealed both conservation and changes of gene function. Here, we study evolutionary changes of lin-39 function using hybrid transgenes and site-directed mutagenesis in an in vivo assay using C. elegans lin-39 mutants. Our data show that despite the functional differences of LIN-39 between the two species, Ppa-LIN-39, when driven by Cel-lin-39 regulatory elements, can functionally replace Cel-lin-39. Furthermore, we show that the MAPK docking and phosphorylation motifs unique for $\mathrm{Cel}$-LIN-39 are dispensable for Cel-lin-39 function. Therefore, the evolution of lin-39 function is driven by changes in regulatory elements rather than changes in the protein itself.
\end{abstract}

[Key Words: Hox genes; evolution; vulva development; Pristionchus pacificus; Caenorhabditis elegans]

Received February 12, 2001; revised version accepted June 22, 2001.

Evolution of animal morphology is ultimately dependent on the evolution of the underlying regulatory genetic networks and of the regulatory genes themselves. Alterations in regulatory genes can either be attributed to changes in expression or to changes in protein structure and function. It has been proposed that changes in the expression of regulatory genes and their downstream targets as caused by mutations in promoter and enhancer regions have played an important role during the evolution of morphological structures (for review, see Tautz 2000). At the same time, evolution of the function of the regulatory proteins themselves is less documented (Golding and Dean 1998; Grenier and Carroll 2000). In fact, there is more evidence for a high conservation of the function of regulatory proteins, even over vast evolutionary distances, often despite a relatively low con-

\footnotetext{
${ }^{1}$ Present address: Aventis Pharma, Fraunhoferstrasse 13, D-82152 Martinsried, Germany.

${ }^{2}$ Corresponding author.

E-MAIL ralf.sommer@tuebingen.mpg.de; FAX 49-7071-601498.

Article and publication are at http://www.genesdev.org/cgi/doi/10.1101/ $\operatorname{gad} .200601$.
}

servation of primary sequences. However, as morphological characters cannot be homologized over large evolutionary distances, a functional comparison often suffers from the difficulty in detecting subtle alterations in gene function.

Nematodes offer an attractive model system for evolutionary studies on animal development because many morphological and cellular structures can easily be homologized between different species (Sommer 2000). Furthermore, the invariant cell lineage of most free-living nematodes allows the study of many morphological alterations at a cellular level. In addition, the wealth of cellular, genetic, and molecular knowledge accumulated for the model organism Caenorhabditis elegans provides an excellent platform for comparative studies (The $C$. elegans Sequencing Consortium 1998). A morphological structure of nematodes that is especially attractive for evolutionary studies is the vulva, the egg-laying and mating structure of nematode females or hermaphrodites. Vulva development has been studied in great detail in C. elegans because it is easily amenable to observation and manipulation at the cellular level (for review, see Greenwald 1997). 
The C. elegans vulva is derived from a subset of 12 ventral epidermal cells, called P1.p-P12.p (Fig. 1A; Sulston and Horvitz 1977). The six central cells, P(3-8).p, are competent to form vulval tissue and constitute the vulval equivalence group. In contrast, the cells in the anterior and posterior body region, $\mathrm{P}(1,2,9-11) \cdot \mathrm{p}$, fuse with the surrounding hypodermal syncytium hyp 7 during the first larval (L1) stage (Fig. 1B). During normal development, the cells of the vulval equivalence group adopt one of three different cell fates. $\mathrm{P}(5-7) . \mathrm{p}$ are induced to form vulval tissue by a signal from the gonadal anchor cell (AC) (Kimble 1981). The central cell P6.p has the $1^{\circ}$ cell fate, generates eight progeny, and forms the inner part of the vulva. $\mathrm{P}(5,7) \cdot \mathrm{p}$ have the $2^{\circ}$ fate, giving rise to seven progeny each, which form the outer part of the vulva. The 22 descendants of $\mathrm{P}(5-7) \cdot \mathrm{p}$ cells will eventually undergo a complex morphogenesis to form the adult vulva. $\mathrm{P}(3,4,8)$.p will, under wild-type conditions, adopt the $3^{\circ}$ cell fate; they divide once and subse- quently fuse with hyp7. Cell ablation studies have indicated that cells that would normally adopt a $2^{\circ}$ or $3^{\circ}$ fate can adopt a higher fate when the cells that normally adopt these fates are eliminated (Sternberg and Horvitz 1986). The six initially equipotent cells $\mathrm{P}(3-8) . p$ therefore have a hierarchical and invariant cell fate pattern $3^{\circ}-3^{\circ}-2^{\circ}-1^{\circ}-2^{\circ}-3^{\circ}$.

Genetic and molecular studies indicate that several different signaling systems interact during vulva formation in C. elegans (Greenwald 1997). The inductive signal from the $\mathrm{AC}$ is a secreted epidermal growth-factorlike molecule encoded by the gene lin-3 (Hill and Sternberg 1992). The EGF signal is transmitted within the vulval precursor cells (VPCs) by EGFR-RAS-MAPK signaling and changes the activity of several transcription factors, including the ETS protein LIN-1 (Beitel et al. 1995), the FKH-like molecule LIN-31 (Tan et al. 1998), and the Hox protein LIN-39 (Maloof and Kenyon 1998).
Figure 1. Summary of ventral epidermal cell fate specification in C. elegans (Cel) and P. pacificus (Ppa) and sequence comparison of Cel-LIN-39 and Ppa-LIN39. (A) Schematic representation of the 12 ventral epidermal Pn.p cells in a C. elegans L1 stage larva. (B) In $C$. elegans (left panel), lin-39 activity defines the $\mathrm{P}(3-8) \cdot \mathrm{p}$ cells as the vulval equivalence group. P6.p (black oval) has the $1^{\circ}$ fate and forms the inner part of the vulva, whereas $\mathrm{P}(5,7) \cdot \mathrm{p}$ (hatched ovals) have the $2^{\circ}$ fate and form the outer part of the vulva. $\mathrm{P}(3,4,8) \cdot \mathrm{p}$ (diagonally striped ovals) remain epidermal and have the $3^{\circ}$ fate. $\mathrm{P}(5-7) . \mathrm{p}$ are induced by the anchor cell (AC) to undergo vulval differentiation. The inductive signal from the AC is encoded by an EGF-like molecule and is transmitted through an EGFR/RAS/MAPK signaling pathway, which modulates the activity of several transcription factors, including LIN-39. In the absence of lin-39 activity (Cel-lin-39), the $\mathrm{P}(3-8) \cdot \mathrm{p}$ cells, like the other Pn.p cells, fuse with the surrounding hypodermis (white ovals). If lin-39 activity is provided during the early step but not during AC induction (Cel hs::1in39 early), the $\mathrm{P}(3-8)$.p cells take on the $3^{\circ}$ fate. In $P$. pacificus (right panel), lin-39 defines $\mathrm{P}(5-8) \cdot \mathrm{p}$ as the vulval equivalence group. $\mathrm{P}(5-7) \cdot \mathrm{p}$ form the vulva with a $2^{\circ}$ $1^{\circ}-2^{\circ}$ fate pattern. P8.p has a special fate designated as $4^{\circ}$ (filled striped oval). Cells not receiving lin-39 activity (Ppa-lin-39) undergo programmed cell death (X). If programmed cell death is inhibited, a normal vulva is formed, indicating that lin-39 is dispensable for vulval formation (Ppa-lin-39; ced-3). It is not known if the $\mathrm{EGF} / \mathrm{RAS} / \mathrm{MAPK}$ pathway is involved in vulval induction by the gonad in $P$. pacificus. $(C)$ Sequence alignment of Cel-LIN-39 and Ppa-LIN-39. The highly conserved homeodomain and hexapeptide motifs are overlined by a solid and a hatched bar, respectively. The putative phosphorylation site in Cel-LIN-39 is indicated by S/TP, and the motif similar to a MAPK docking site (Jacobs et al. 1999) is indicated by FXFP. Note that these motifs are absent in Ppa-LIN-39. (D) Schematic representation of the LIN-39 primary structure. (Hex) Hexapeptide motif, (HD) homeodomain. Solid and open arrowheads above the bar indicate the positions of all published nonsense and missense mutations, respectively, found in C. elegans lin-39 alleles (Clark et al. 1993; Wang et al. 1993). Arrows indicate nonsense mutations introduced in C. elegans rescue constructs. Solid arrowheads below the bar indicate all nonsense mutations found in P. pacificus lin-39 alleles (Eizinger and Sommer 1997).

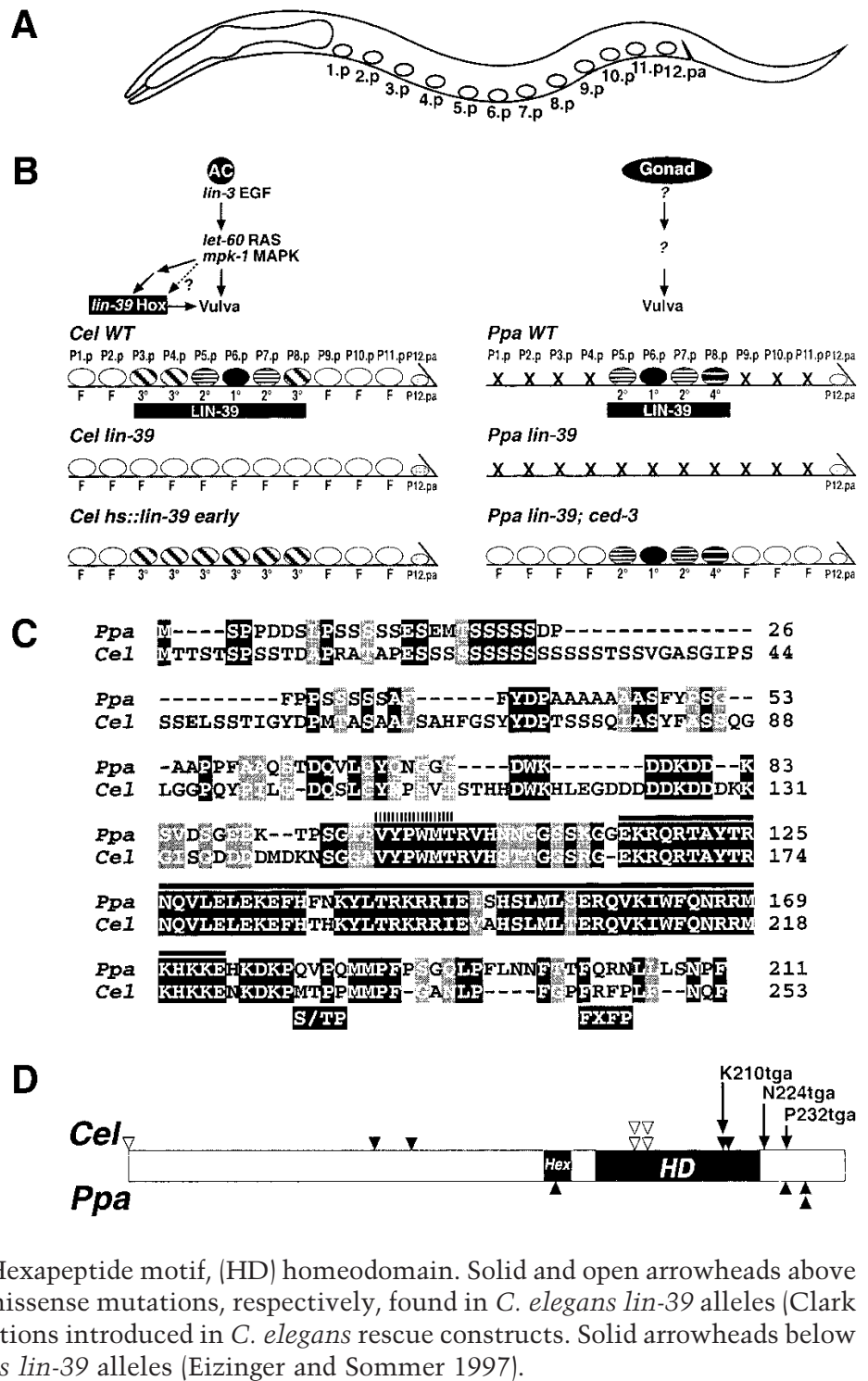


LIN-39 has been shown to play a role at two stages during C. elegans vulval development (Clark et al. 1993; Wang et al. 1993; Clandinin et al. 1997; Maloof and Kenyon 1998). First, during the early phase of vulval development, lin-39 expression is required to prevent the VPCs, $\mathrm{P}(3-8) . p$, from cell fusion. In Cel-lin-39 mutants, $\mathrm{P}(3-8) . p$ fuse with hyp7, like their anterior and posterior counterparts (Fig. 1B). Second, expression of lin-39 is also crucial at a later stage during vulva induction: If LIN-39 is provided early in larval development but not during vulval induction, all VPCs have a $3^{\circ}$ fate (Fig. 1B, Cel hs::lin-39 early). It has been shown that lin-39 expression levels are up-regulated in $\mathrm{P}(5-7)$.p dependent on EGF signaling from the AC and an additional Wnt signaling activity (Eisenmann et al. 1998; Maloof and Kenyon 1998). Also, it has been speculated that LIN-39 might be a direct target for MAPK phosphorylation (Fig. 1B; Eisenmann et al. 1998).

The nematode Pristionchus pacificus has been established as a satellite organism to C. elegans for evolutionary comparative studies (Sommer and Sternberg 1996; Sommer et al. 1996). In particular, studies of vulval development in $P$. pacificus have revealed several differences at the cellular and genetic levels between the two nematodes (for review, see Eizinger et al. 1999). The ventral epidermal cells that are not competent to form vulval tissue in $P$. pacificus do not fuse with the surrounding hypodermis, as in C. elegans, but instead undergo programmed cell death. In addition, vulval competence is restricted to a small subset of ventral epidermal cells, because only $\mathrm{P}(5-8) \cdot \mathrm{p}$ instead of $\mathrm{P}(3-8) \cdot \mathrm{p}$ are part of the vulval equivalence group (Fig. 1B). Genetic studies in $P$. pacificus revealed that the role of lin-39 has changed during evolution. In Ppa-lin-39 mutants, P(5-8).p undergo programmed cell death, like their anterior and posterior neighbors (Fig. 1B; Eizinger and Sommer 1997). Therefore, in P. pacificus, lin-39 prevents the VPCs from undergoing cell death instead of preventing fusion as in C. elegans. In addition, unlike Cel-lin-39, which is required twice during vulval development, Ppa-lin-39 is only required during the first step, but is dispensable for vulval induction. This was demonstrated by generating double mutants that are deficient for both lin-39 and cell death activity and are able to form a normal vulva (Fig. 1B, Ppa-lin-39; ced-3; Sommer et al. 1998).

The described differences in lin-39 gene function between $C$. elegans and $P$. pacificus offer an interesting evolutionary framework to compare, at the molecular level, the role of an important regulatory gene in homologous cellular and developmental contexts. Is the evolution of lin-39 function accompanied by changes in the regulatory elements of the lin-39 gene and/or changes of the coding region itself? How important are specific motifs of the LIN-39 protein? We have addressed these questions by introducing Ppa-lin-39 in a Cel-lin39 mutant background, under the control of either Ppalin-39 regulatory elements or regulatory regions from the Cel-lin-39 gene itself. In addition, we have addressed the possible role of Cel-LIN-39 as a direct target for MAPK phosphorylation. Our data show that despite the differ- ent roles of LIN-39 in C. elegans and P. pacificus, PpaLIN-39, when expressed under the control of Cel-lin-39 regulatory elements, can functionally replace Cel-lin-39 and rescue a strong Cel-lin-39 mutant. Furthermore, we show that unique putative MAPK docking and phosphorylation sites of Cel-LIN-39 are dispensable for CelLIN-39 function during vulval development.

\section{Results}

Sequence comparison of $\mathrm{C}$. elegans and $\mathrm{P}$. pacificus LIN-39

The role of lin-39 during vulval development differs between C. elegans and P. pacificus in several aspects (Fig. 1B; Eizinger et al. 1999|. First, in C. elegans, lin-39 acts to prevent the VPCs from cell fusion, whereas in $P$. pacificus, the corresponding cells are protected from programmed cell death. Second, the vulval equivalence group is smaller in $P$. pacificus than in C. elegans, only consisting of $\mathrm{P}(5-8) . p$ instead of $\mathrm{P}(3-8) . p$. Third, Cel-lin39 plays two separate roles during vulval development, early to establish the equivalence group and later during vulval induction (Clandinin et al. 1997; Maloof and Kenyon 1998). In contrast, Ppa-lin-39 is only required during the first step (Sommer et al. 1998). These differences suggest that the function of lin-39 might have changed at the molecular level. These alterations might include changes in expression, altered downstream targets, and/or intrinsic changes in the LIN-39 protein itself. Comparison of the primary structures of Cel-LIN39 and Ppa-LIN-39 shows a high degree of sequence conservation in the hexapeptide and homeodomain regions required for DNA-binding (Fig. 1C). However, in the Nterminal and C-terminal domains, sequence similarity is considerably lower. Given the differences between Ppalin-39 and Cel-lin-39 function and sequence, we wondered whether the altered role of lin-39 during vulval development might correlate with changed properties of the LIN-39 proteins.

Furthermore, upon inspection of the inferred amino acid sequence of the Cel-LIN-39 primary sequence, we found that the C-terminal domain contains a close match to a consensus MAPK docking and phosphorylation sequence (F-X-F-P and S/T-P, respectively; Fig. 1C). A MAPK docking site of similar sequence was previously identified in other C. elegans proteins (Jacobs et al. 1999|. The presence of a single putative MAPK docking and phosphorylation site suggested that Cel-LIN-39 might be a direct target for MAPK phosphorylation. Interestingly, this MAPK target sequence is not conserved in Ppa-LIN-39 and might therefore be responsible for the differences between the roles of lin-39 in the two species.

The C. elegans lin-39 gene contains multiple regulatory regions

Given the differences in lin-39 function between C. elegans and P. pacificus, as well as the limited lin-39 se- 
quence conservation, we wanted to address the question, can Ppa-lin-39 functionally replace Cel-lin-39? Previous work toward a functional comparison of Cel-lin-39 with Hox proteins from other species has been based on overexpression studies using a heat-shock promoter (Hunter and Kenyon 1996). To establish a more sensitive system for the detection of possible differences in LIN-39 function, we decided to use the regulatory elements from the Cel-lin-39 gene itself to drive expression of Ppa-lin-39 in C. elegans. For this purpose, we identified the regulatory regions of the Cel-lin-39 gene by generating a series of LacZ reporter constructs. These constructs are based on a 10-kb genomic region that covers the Cel-lin-39 gene (Fig. 2A).

The expression patterns generated by these constructs were analyzed by studying several transgenic lines in a C. elegans wild-type background. In pKG1 transgenic animals, containing the entire 10-kb region, lin-39 expression was observed in the ventral midbody region in embryos and larvae and in the vulval muscle cells of adult worms (Fig. 2B). This expression pattern was identical to the previously reported Cel-LIN-39 antibody staining (Maloof and Kenyon 1998) and therefore suggested that this fragment contains the regulatory elements required to drive lin-39 expression (Fig. 2B). Deletion of the entire promoter region (pKG2) did not abolish expression but gave a strong ectopic expression pattern in embryos and larvae (Fig. 2B). However, weak expression was still observed in adult vulval muscles, suggesting that this latter expression is under control of regulatory elements downstream of the transcription start site.

To determine if the Cel-lin-39 gene contained intronic regulatory elements, the introns were removed from the reporter construct (pKG3). In pKG3 transgenic animals, no expression was observed in adult worms but ectopic expression was seen in embryonic and larval stages in various parts of the body (Fig. 2A). Interestingly, most of the normal expression could be restored by adding back the second intron of Cel-lin-39 (pKG4), which suggests that this intron contains important regulatory elements. When only the promoter region was fused to a LacZ reporter vector, a strong ectopic posterior expression was observed (pKG5; Fig. 2A,B). Surprisingly, replacing the 3' UTR of pKG5, which is derived from the unc-54 gene, with the 3' UTR from the Cel-lin-39 gene (pKG6), partially restored the normal expression pattern (Fig. 2A). This result suggests that the 3' UTR of the Cel-lin-39 gene is important for correct expression, which could be either at the transcriptional or the posttranscriptional level.

We also asked if the regulatory regions of the Ppa-lin39 gene could be used to drive reporter gene expression in C. elegans. However, no LacZ staining was observed when a 12-kb genomic region spanning the Ppa-lin-39 gene was used to generate transgenic $C$. elegans lines (pKG7; Fig. 2A). This result is in agreement with the lack of significant conservation of noncoding regions in the C. elegans and Ppa-lin-39 genes (data not shown), suggesting that the regulatory elements may have evolved
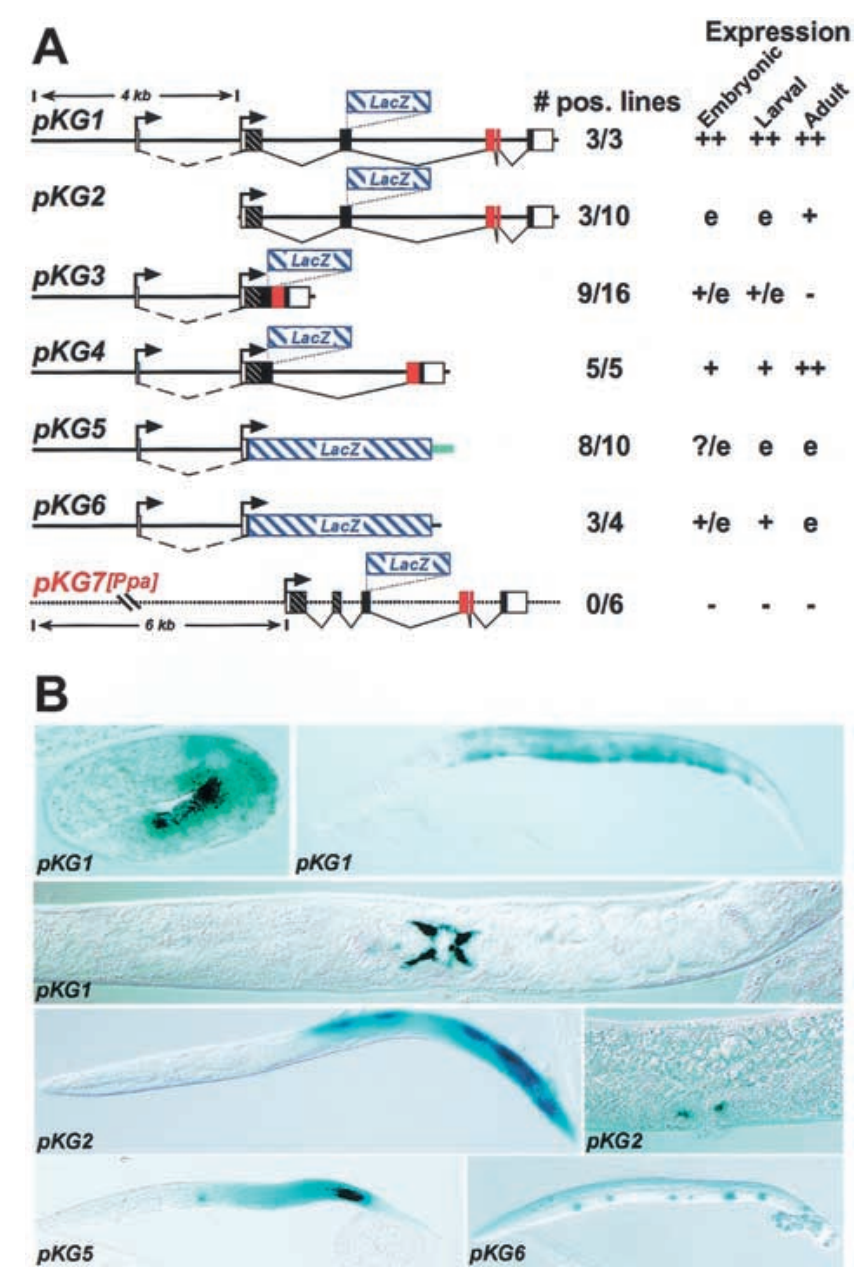

Figure 2. Characterization of regulatory regions in the C. elegans lin-39 gene by the use of LacZ reporter constructs. (A) Schematic description of the LacZ reporter constructs analyzed and the obtained expression patterns. Rectangles indicate lin-39 exons; (red) homeodomain-encoding exons; (filled and hatched) other coding exons; (open) $5^{\prime}$ and $3^{\prime}$ untranslated exons. The green bar indicates the unc-54 3' UTR in pKG5. The LacZ reporter gene is indicated by a blue, hatched rectangle. Bent arrows represent two putative transcription start sites. Note that pKG7 is based on the P. pacificus lin-39 gene. For each construct at least three transgenic lines were investigated. $(++)$ Apparently normal expression; $(+)$ weaker than normal expression; (e) ectopic expression; (-) no expression. (B) Representative examples of expression patterns obtained for the constructs pKG1, pKG2, pKG5, and KG6. See text for detailed description.

considerably during the approximately 200 million years that separate these two nematode species.

Rescue analysis reveals the requirement for more distal genomic regions

To verify that the 10-kb genomic region of the Cel-lin-39 gene contained all regulatory elements necessary for correct lin-39 expression, we tested if this genomic fragment was sufficient to rescue a strong lin-39 loss-of- 
function allele, lin-39(n1880), when introduced as a transgene. We assayed the degree of rescue by counting animals positive for egg-laying. Indeed, a construct containing the 10-kb genomic region of the Cel-lin-39 gene (pKG8) was sufficient for rescue but with a low frequency (Fig. 3). Specifically, in all five transgenic lines tested, only about $15 \%$ of the animals were egg laying positive (Fig. 3A).

To determine if the use of a larger genomic region would increase the rescuing activity, we generated transgenic lines using a $23-\mathrm{kb}$ genomic clone, which virtually spans the entire lin-39 locus between the two neighboring genes (pKG9). Using this construct, rescue of the egglaying defect was increased more than threefold to $54 \%$ in all tested transgenic lines (Fig. 3A). This result suggests that although the $10-\mathrm{kb}$ fragment contains the most important regulatory regions, as can be defined by studying LacZ expression, there are additional regulatory regions located more upstream and/or downstream. An alternative explanation could be that the addition of more $5^{\prime}$ and $3^{\prime}$ regions renders the transgenic construct a more stable chromatin structure, which in turn improves lin-39 expression.

We also asked whether the Ppa-lin-39 gene could rescue the egg-laying defect of lin-39(n1880) using the same $12-\mathrm{kb}$ genomic fragment previously analyzed for LacZ expression. However, this fragment (pKG10) was unable to rescue Cel-lin-39(n1880) (Fig. 3A).

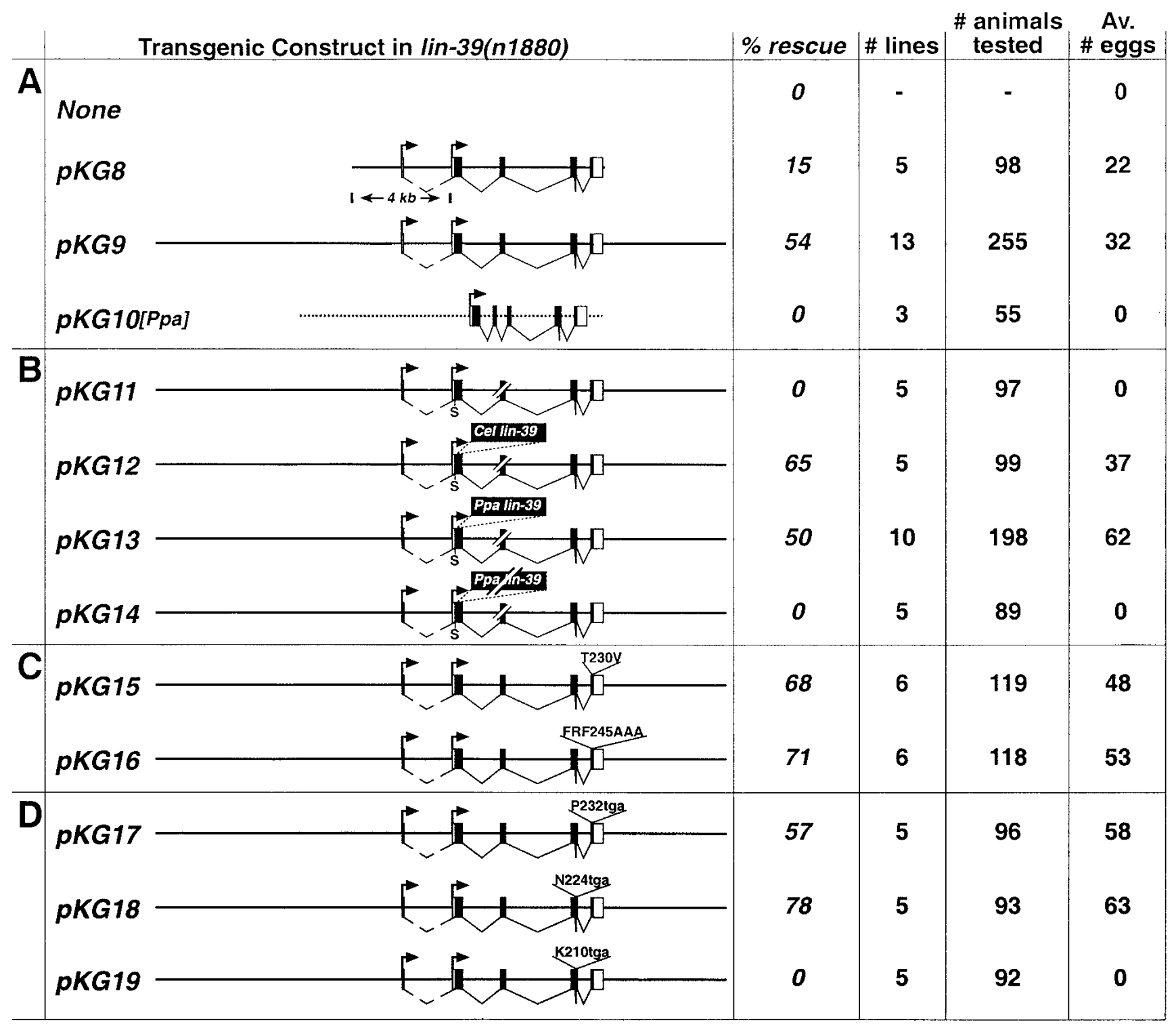

Figure 3. Summary of egg-laying rescue experiments. All transgenic constructs were analyzed in the strain MT4498 carrying the null allele Cel-lin-39(n1880), which is completely egg-laying defective. At least three transgenic lines were tested for each construct. (Filled rectangles) Coding exons; (open rectangles) 5' and 3' noncoding exons; (bent arrows) transcription start sites; (S) the unique restriction site in pKG11-pKG14; (diagonal bars) introduced frame-shift mutations. Inserted cDNAs in pKG12-pKG14 are indicated. 
Rescue of the Cel-lin-39 egg-laying phenotype by Ppa-LIN-39 expression

To determine if the evolution of lin-39 function is driven by changes in the regulatory elements and/or changes in the coding region, we analyzed the rescuing activity of Ppa-lin-39 transgenes in Cel-lin-39 mutants. The LacZ expression studies and the rescue experiments indicate that the expression of Ppa-lin-39 as a transgene in C. elegans, similar to the endogenous Cel-lin-39 gene, requires the use of multiple Cel-lin-39 regulatory elements distributed over a large genomic interval. Therefore, we generated an expression cassette based on the $23-\mathrm{kb}$ construct of pKG9, by introducing a unique restriction site just downstream of the endogenous Cellin-39 translation start site, in which a complete cDNA can be inserted (Fig. 3B). As this construct retained all exons of the Cel-lin-39 gene from which a functional Cel-LIN-39 protein could be generated, we additionally introduced a frame-shift mutation in the second exon. The resulting construct pKG11 did not rescue the lin-39 egg-laying defect (Fig. 3B). However, by introducing a Cel-lin-39 cDNA into this expression cassette, rescue could be restored completely (pKG12). Of the transgenic animals 65\% were egg laying positive, demonstrating the functionality of the expression system (Fig. 3B).

To analyze if the functional differences between Cellin-39 and Ppa-lin-39 result from differences in the coding region, we substituted the Cel-lin-39 cDNA with a cDNA encoding Ppa-lin-39 (pKG13). This construct also rescued the egg-laying defect when analyzed in transgenic Cel-lin-39(n1880) animals (Fig. 3B). In particular, almost half of the transgenic animals carrying the pKG13 construct were egg laying positive (Fig. 3B). To verify that the rescue activity was dependent on the inserted Ppa-lin-39 cDNA, we disrupted the coding region of the inserted Ppa-lin-39 cDNA by a frame-shift mutation (pKG14). pKG14 has no rescuing activity, indicating that egg laying, as observed in pKG13 transgenic animals, was dependent on the Ppa-lin-39 cDNA (Fig. 3B).

Restoration of the C. elegans vulval lineage by Ppa-LIN-39 expression

Next, we asked whether the vulval defects of Cel-lin39(n1880) were completely rescued at a cellular level in pKG13 transgenic animals. In Cel-lin-39(n1880) mutant animals, $\mathrm{P}(4-8) . \mathrm{p}$ are not prevented from fusing with hyp7 early in larval development so that the fate of all five cells differs from wild-type animals (Fig. 4A). We analyzed the vulval cell lineage in 11 Cel-lin-39(n1880) pKG13 transgenic animals and observed both complete and partial rescue of the vulval cell lineages (Fig. 4). In total, 33 of $55(60 \%)$ of the VPCs did not fuse with hyp 7 . However, among the VPCs that did fuse, we observed a strong positional bias: more than $70 \%$ of these VPCs were peripheral cells, P4.p or P8.p, respectively. In contrast, the central cell P6.p did not fuse and had a $1^{\circ}$ fate in all animals analyzed.

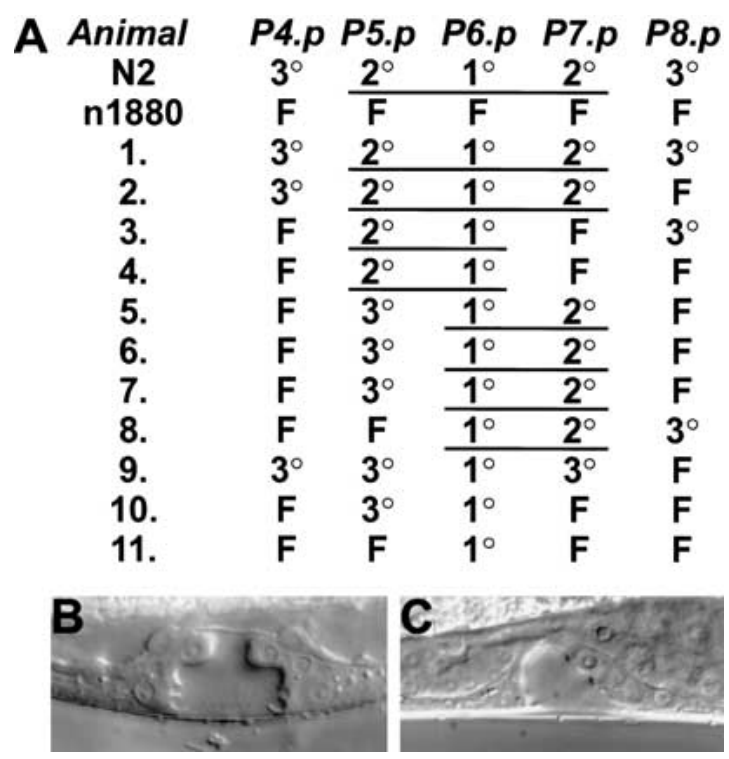

Figure 4. Vulval cell fate in Cel-lin-39(n1880) animals rescued by Ppa-lin-39 expression. (A) Vulval cell fate patterns obtained by Nomarski microscopy for 11 worms transgenic for the construct pKG13. $1^{\circ}, 2^{\circ}, 3^{\circ}$, and $\mathrm{F}$ fates are defined in Figure 1. Underlined cells form one continuous vulval invagination. Vulval cell fates for wild-type (N2) and lin-39(n1880) animals are shown as reference. $(B)$ Example of completely rescued vulval cell fates in the L4 larval stage. $(C)$ Example of partially rescued vulval cell fates with one $1^{\circ}$ and one $2^{\circ}$ cell in the L4 larval stage.

The wild-type vulva is formed by the progeny of $\mathrm{P}$ (57).p with a $2^{\circ}-1^{\circ}-2^{\circ}$ cell fate pattern. In pKG 13 transgenic animals, 6 out of 33 of these central VPCs (18\%) underwent cell fusion, whereas another $18 \%$ of these cells had a $3^{\circ}$ fate; 10 out of $22(45 \%) \mathrm{P}(5,7) . p$ cells had a $2^{\circ}$ fate; and $100 \%$ of the P6.p cells had a $1^{\circ}$ fate (Fig. 4A,B). Therefore, the rescuing activity is about $60 \%$ for both the fusion versus nonfusion and the vulval $\left(1^{\circ} / 2^{\circ}\right.$ fate $)$ versus nonvulval $\left(3^{\circ}\right)$ fate decision. These results suggest that Ppa-lin-39 can rescue both functions of Cel-lin-39, the formation of the vulva equivalence group (fusion vs. nonfusion) and the later function as a component of EGF/RAS/MAPK signaling (vulval vs. nonvulval). However, they are not consistent with the absence of the MAPK docking and phosphorylation motifs being responsible for the different functions of lin-39 in both species.

\section{Rescue of VC neurons by Ppa-LIN-39 expression}

Another function of LIN-39 in C. elegans is the formation of the VC neurons. The six VC neurons of C. elegans are generated as a subset of the Pn.aap cells, which derive from the Pn.a neuroblasts, the sister cells of the Pn.p ectoblasts (Fig. 5; Sulston and Horvitz 1977). The fate of the Pn.aap cells is position-dependent and relies on lin39 function. $\mathrm{P}(3-8)$.aap normally form VC neurons, whereas in lin-39 mutants these cells instead undergo programmed cell death, like their anterior and posterior 

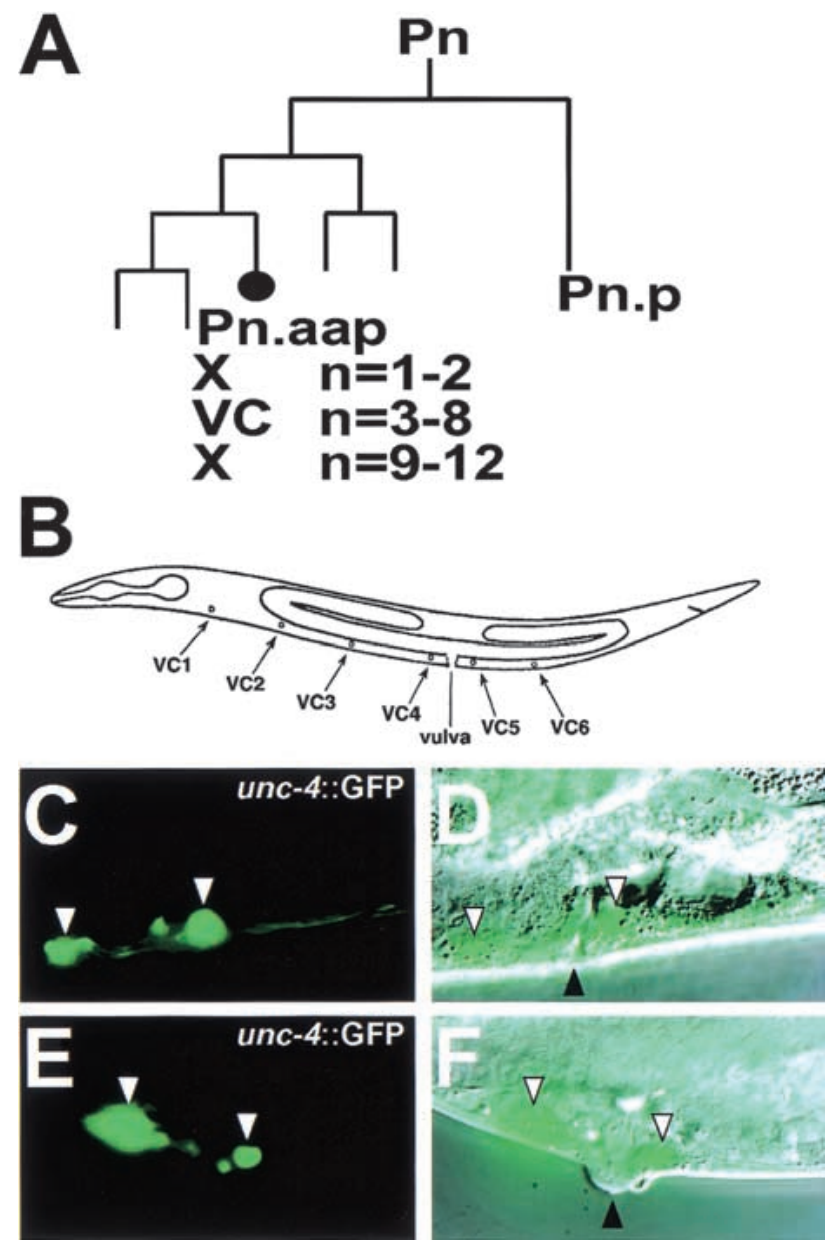

Figure 5. Rescue of the fate of the VC neurons in Cel-lin39(n1880) by Ppa-lin-39 expression. Schematic representation of the $\mathrm{P}$ cell lineage with $n$ denoting cell number. In wild-type C. elegans, the $\mathrm{P}(3-8)$.aap cells form VC neurons, whereas $\mathrm{P}(1,2,9-12)$.aap cells undergo programmed cell death $(\mathrm{X})$. (B) Schematic representation of the positions of the six VC neurons. Note especially the positions of VC4 and VC5 in close proximity to the vulva (data not shown). $(C-F)$ Expression of unc-4::GFP in VC4 and VC5 neurons in lin-39(n1880) animals rescued by $P$. pacificus lin-39 expression. No VC neurons are formed in lin-39(n1880) mutant animals. $(C, E)$ GFP expression. Open arrowheads indicate GFP expressing cells. $(D, F)$ The same pictures as in $\mathrm{C}$ and $\mathrm{E}$, respectively, overlaid with the corresponding Nomarski pictures. Filled arrowheads indicate the vulva.

lineage homologs, $\mathrm{P}(1,2,9-12)$.aap (Clark et al. 1993; Salser et al. 1993). In P. pacificus, only four VC neurons are formed by $\mathrm{P}(5-8)$.aap, whereas $\mathrm{P}(1-4,9-12)$.aap die of programmed cell death (Eizinger et al. 1999). In Ppa-lin39 mutant animals, no VC neurons are formed and $\mathrm{P}(5-$ 8).aap die like their anterior and posterior lineage homologs, indicating that lin-39 might have a similar function during VC neuron formation in both species (Eizinger et al. 1999).

Cell ablation studies in C. elegans indicated that VC neurons are dispensable for egg laying ( $\mathrm{Li}$ and Chalfie
1990). Therefore, the egg-laying assay is unreliable to check whether Ppa-lin-39 expression can rescue C. elegans VC neurons from programmed cell death. We asked whether VC neurons were present in Cel-lin-39(n1880) mutant animals expressing Ppa-lin-39 by generating transgenic lines carrying the Ppa-lin-39 expression vector pKG13 and unc-4::GFP, a marker for several different neurons in the ventral midline, including the VC neurons (Miller and Niemeyer 1995; Pflugrad et al. 1997; Lickteig et al. 2001). In the transgenic lines carrying pKG13 and unc-4::GFP, we observed expression of the GFP marker in the proximity of the adult vulva, indicating the presence of the VC4 and VC5 cells (Fig. 5B). These results suggest that expression of $P p a$-LIN-39 can rescue the C. elegans VC neurons from undergoing programmed cell death and that Ppa-LIN-39 can replace Cel-LIN-39 function not only in vulval, but also in neuronal cell lineages.

\section{The MAPK phosphorylation site in Cel-LIN-39 is dispensable for vulva formation}

Given the observation that Cel-LIN-39 contains a putative MAPK target site and expression levels are up-regulated by EGF/RAS/MAPK signaling, LIN-39 might be a direct target of MAPK phosphorylation (Eisenmann et al. 1998). However, the observation that Ppa-LIN-39 expression can functionally replace Cel-LIN-39 argues against LIN-39 being a direct target of MAPK because the putative phosphorylation site and MAPK docking site are absent in Ppa-LIN-39 (Fig. 1C). To study further whether phosphorylation of Cel-LIN-39 is of importance for LIN-39 function during vulva development, we generated point mutations in Cel-lin-39 by site-directed mutagenesis (Fig. 3C). The two constructs pKG15 and pKG16 lead to expression of a LIN-39 protein lacking the putative phosphorylation site (T230V) or the consensus MAPK docking site (FRF245AAA), respectively. Both of these mutated forms of Cel-LIN-39 rescued the egg-laying defect of $n 1880$ to a similar degree as the Cel-lin-39 or Ppa-lin-39 wild-type cDNAs (Fig. 3C). These results suggest that MAPK phosphorylation is dispensable for LIN-39 function during vulval development. However, these experiments do not rule out the possibility that phosphorylation of Cel-LIN-39 might play a role in other developmental processes.

\section{Cel-LIN-39 is not phosphorylated by MAPK in vitro}

Next, we asked whether Cel-LIN-39 is a substrate for MAPK phosphorylation in vitro. Wild-type LIN-39 and LIN-39(T230V) were translated in vitro to generate either unlabeled or ${ }^{35}$ S-labeled proteins. Human Elk-1, a known target for MAPK phosphorylation (Janknecht et al. 1993; Yang et al. 1998), was used as control. As expected, Elk-1 clearly demonstrated MAPK-dependent phosphorylation; ${ }^{35}$ S-labeled Elk-1 showed a slower migration after phosphorylation (Fig. 6, cf. lanes 9 and 12), and unlabeled protein showed a MAPK-dependent phos- 


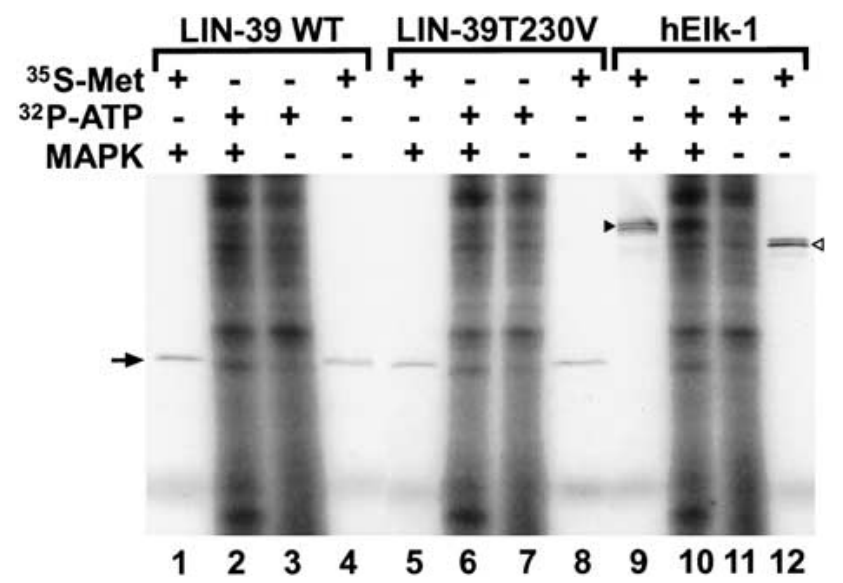

Figure 6. In vitro MAPK phosphorylation. Wild-type or phosphorylation-defective (T230V) Cel-LIN-39 as well as human Elk-1 were generated by in vitro translation in the presence of ${ }^{35}$ S-methionine (lanes $1,4,5,8,9,12$ ) or unlabeled methionine (lanes 2,3,6,7,10,11) and were subsequently used for in vitro phosphorylation by $\mathrm{p} 42$ MAPK. Unlabeled proteins were phosphorylated with $\left[\gamma^{-32} \mathrm{P}\right] \mathrm{ATP}$, leading to MAPK-dependent and MAPK-independent labeling of many proteins in the in vitro translation reaction (lanes 2,3,6,7,10,11). Proteins labeled with ${ }^{35} \mathrm{~S}$ were phosphorylated with nonradioactive ATP. Phosphorylation of hElk-1 was readily observed; note the difference in migration (lanes 9,12) for ${ }^{35} \mathrm{~S}$-labeled protein and the increase in $\left[\gamma^{-32} \mathrm{P}\right]$ ATP-dependent phosphorylation (lanes 10,11). In contrast, no sign of phosphorylation of Cel-LIN-39 could be observed (cf. lanes 1 and 4, 2 and 3). Note that a protein of slightly faster mobility than Cel-LIN-39 shows a partially MAPK-dependent phosphorylation (cf. lanes 2 and 3, 6 and 7, 11 and 12). An arrow indicates the size of Cel-LIN-39, a filled arrowhead phosphorylated hElk-1, and an open arrowhead unphosphorylated hElk-1, respectively.

phorylation with $\left[\gamma^{-32} \mathrm{P}\right] \mathrm{ATP}$ (Fig. 6, lanes 10,11). In contrast, wild-type Cel-LIN-39 was not phosphorylated; no shift in migration was observed for ${ }^{35} \mathrm{~S}$-labeled protein in the presence of MAPK (Fig. 6, lanes 1,4), and incubation of unlabeled Cel-LIN-39 with $\left[\gamma^{-32}{ }^{3}\right]$ ATP did not yield any detectable change in phosphorylation (Fig. 6, lanes 2,3). Phosphorylation-defective Cel-LIN-39(T230V) behaved identically to wild-type Cel-LIN-39 (Fig. 6, lanes 5-8). Therefore, the in vitro phosphorylation experiments suggest that Cel-LIN-39 cannot act as a direct target for MAPK, in spite of the presence of the conserved MAPK-docking and phosphorylation sequences.

The C terminus is dispensable for LIN-39 function in C. elegans vulval development

The mutational analysis of the LIN-39 C terminus suggested that Cel-LIN-39 function might be relatively indifferent to mutations in the $\mathrm{C}$ terminus (Figs. 1D, 3C). This is in agreement with the distribution of molecular lesions in genetically isolated Cel-lin-39 mutants. Of the many missense and nonsense mutations described for Cel-lin-39, none is located in the C terminus, suggesting that mutations in this region would not be de- tected in a genetic screen (Fig. 1D; Clark et al. 1993; Wang et al. 1993). This is in contrast to the molecularly characterized Ppa-lin-39 alleles, where three out of four mutations are located in the C terminus (Fig. 1D; Eizinger and Sommer 1997). Also, the extent of VPC survival and vulva differentiation differs among the $P p a-$ lin-39 alleles with molecular lesions in the C-terminal region. Ppa-lin-39(tu2) contains a nonsense mutation resulting in a stop codon at amino acid position 183 and causes a severe vulval phenotype. In contrast, Ppa-lin39(tu29) contains a nonsense mutation with a stop codon at amino acid position 192, resulting in a protein that is only nine amino acids longer than the one in Ppa-lin-39(tu2), but causes only a mild vulval phenotype (Eizinger and Sommer 1997). Although we cannot completely rule out the possibility that the differences between tu2 and tu29 might be owing to a nonsense-mediated decay, we speculate that the C-terminal domain of LIN-39 plays an important function in Ppa-LIN-39, whereas it might be of less importance for Cel-LIN-39 function.

To determine the role of the C-terminal region of Cellin-39, we generated C-terminal nonsense mutations and tested these constructs for transgenic rescue of the egglaying defect (Fig. 3D). The first construct tested, pKG17 (P232tga), encodes a protein lacking the last 22 amino acids, leaving only 8 amino acids C-terminal to the homeodomain, which is comparable to Ppa-lin-39(tu2). However, pKG17 (P232tga) could rescue the egg-laying defect of Cel-lin-39(n1880) to the same extent as the wild-type construct pKG8 (Fig. 3D). Even the deletion of the remaining amino acids $\mathrm{C}$-terminal to the homeodomain (pKG18, N224tga) did not impair lin-39 function (Fig. 3D). Therefore, Cel-LIN-39 completely lacking the $\mathrm{C}$-terminal domain remains functional in vulva development. In contrast, introduction of a stop codon 14 amino acids more upstream (pKG19, K210tga) completely abolished lin-39 rescuing activity (Fig. 3D). This result was expected because the K210tga mutation disrupts the homeodomain and furthermore corresponds to a genetically isolated null allele Cel-lin-39(n1760) (Clark et al. 1993).

\section{Discussion}

Previous investigations of vulval development in $P$. pacificus, at the cellular and genetic levels, have indicated several differences in the function of the Hox gene lin-39 between this nematode and C. elegans (Eizinger and Sommer 1997; Sommer et al. 1998). In this study we have compared the conservation of LIN-39 protein function and lin-39 regulatory regions at the molecular level in these two nematodes. The results suggest that noncoding regulatory regions are not conserved, whereas the protein coding regions, although only partially conserved in sequence, still show a high degree of functional conservation. Nevertheless, functional differences appear to exist, for example, in the importance of the C-terminal domain. In addition, our data argue against the postulated role of the C-terminal domain of Cel-LIN-39 as a 
direct target for MAPK phosphorylation during vulval development.

\section{Expression of the Cel-lin-39 gene is under complex regulatory control}

Our initial experiments were aimed at defining the minimal regulatory units required to drive Cel-lin-39 expression. These experiments demonstrated that Cel-lin-39 is under the control of multiple regulatory regions and suggested a complex interaction between these elements. Only by using a construct containing a large promoter element, the second intron, and the $3^{\prime}$ UTR from the Cel-lin-39 gene was it possible to obtain an expression pattern similar to the protein expression reported previously (Maloof and Kenyon 1998). The corresponding 10$\mathrm{kb}$ genomic fragment also rescued a lin-39 null mutant. However, additional regulatory elements are present in more distal genomic regions, because the use of a $23-\mathrm{kb}$ genomic fragment improved the rescuing activity about threefold.

The complex organization of regulatory units in the Cel-lin-39 gene made it challenging to create a reliable fusion construct in which the Ppa-lin-39 cDNA is under the control of the regulatory elements from the Cel-lin39 gene. The approach of introducing a cDNA (with its own stop codon) downstream of the natural translation start site proved successful in this study. It was initially not clear that this technique should work, as it has been reported that mRNAs with a premature stop codon are selectively degraded through nonsense-mediated decay (Pulak and Anderson 1993; Anderson and Kimble 1997). However, because both the Cel-lin-39 cDNA and the Ppa-lin-39 cDNA could rescue a C. elegans lin-39 null allele in this type of construct, it appears that sufficiently high mRNA levels could be obtained.

\section{Changes in the regulatory regions of lin-39}

Expression analysis of a Ppa-lin-39 LacZ reporter construct in C. elegans failed to show any expression (Fig. 2). In addition, the corresponding construct without a LacZ reporter also failed to rescue a Cel-lin-39 mutation (Fig. 3). Together, these results suggest that the requirements of the regulatory regions of the lin-39 gene differ between C. elegans and $P$. pacificus. Regulatory evolution of nematode Hox genes therefore plays an important role for the development and evolution of these organisms and the underlying changes of cellular processes.

Promoter and intronic regions of the lin-39 gene show no detectable conservation between both species (data not shown). Therefore, C. elegans and $P$. pacificus are evolutionarily too far apart to allow a meaningful comparison of noncoding regulatory regions in the lin-39 gene. To allow for such a comparison it will be necessary to obtain and include sequence information from more closely related species. In addition, identification of regulatory elements in the Ppa-lin-39 gene would best be done by introducing transgenic reporter con- structs into P. pacificus, and not into C. elegans. However, it has so far not been possible to establish transgenic technology in P. pacificus (C. Sigrist and R.J. Sommer, unpubl.).

\section{Expression of Ppa-LIN-39 can rescue a Cel-lin-39 mutant}

The finding of nonconservation of regulatory elements between the Ppa-lin-39 and Cel-lin-39 genes is in agreement with most studies in developmental evolution, which specifically emphasize the importance of regulatory interactions for developmental evolution (Tautz 2000). In contrast, studies on the evolution of coding regions are only rarely undertaken in this context, and most of such studies are more concerned with the evolution of codon usage than with the evolution of protein function(s) themselves (for review, see Kreitman and Comeron 1999). Golding and Dean (1998) review studies on metabolic enzymes in which phylogenetics and protein structure and function have been integrated with site-directed mutagenesis, indicating the importance of protein evolution. However, studies on developmental control genes only rarely use site-directed mutagenesis to uncover the importance of individual amino acids. In addition, most of the latter studies are carried out by using overexpression systems but do not use gene-specific promoter/enhancer constructs. In the present study we have tried to combine phylogenetics with protein structure/function aspects in the context of developmental evolution. As the copy number of transgenes cannot be precisely controlled in $C$. elegans rescuing experiments, we tested multiple independent transgenic lines.

The present work indicates that the Ppa-LIN-39 protein can take over the functions of Cel-LIN-39 and rescue a Cel-lin-39(n1880) mutation when expressed under the control of the Cel-lin-39 regulatory elements. Therefore, although Ppa-lin-39 gene functions partially differ from those of Cel-lin-39 when compared at the cellular and genetic levels, the rescue experiments show that at the molecular level, Ppa-LIN-39 is sufficiently conserved to functionally replace Cel-LIN-39. We analyzed the rescue in three different ways: (1) overall rescue of egg laying, (2) restoration of the vulval cell lineage, and (3) rescue of the VC neurons. Although transgenic rescue experiments in C. elegans have to be considered with care, our results indicate that Ppa-LIN-39 restores all of these functions tested. Specifically, the analysis of the vulval cell lineage in animals rescued by Ppa-LIN-39 expression showed that all aspects of the vulval lineage could be restored. This finding is surprising because it includes functions that are not present in the $P$. pacificus vulval lineage itself, like the inhibition of cell fusion in $\mathrm{P}(3-8) . \mathrm{p}$ during the early phase of vulval development and the generation of a $3^{\circ}$ lineage in $\mathrm{P}(4,8)$.p.

Functional difference of the LIN-39 C-terminal domain between $\mathrm{C}$. elegans and $\mathrm{P}$. pacificus

The rescue experiments demonstrated that Ppa-LIN-39 could replace Cel-LIN-39 in all investigated functions. 
Nonetheless, the observed differences in the distribution of mutations in the lin-39 alleles between the two species suggests that there are functional differences between the two LIN-39 proteins. No alleles of Cel-lin-39 contain nonsense or missense mutations in the C-terminal domain, whereas three out of the four Ppa-lin-39 alleles available are nonsense mutations in the $\mathrm{C}$ terminus (Clark et al. 1993; Eizinger and Sommer 1997). This suggests that the $\mathrm{C}$-terminal domain plays an important role in lin-39 function in P. pacificus but that it might be dispensable for Cel-lin-39 activity. Our mutational analysis of the Cel-lin-39 gene supports the latter conclusion because deletion of the entire $\mathrm{C}$ terminus did not affect the rescuing activity of Cel-lin-39. A direct mutational analysis of the Ppa-LIN-39 protein to corroborate these results will have to await the establishment of transgenic technology in $P$. pacificus.

Functional differences between homologous Hox proteins have also been observed while studying the evolution of the Ultrabithorax (Ubx) protein among arthropods. Grenier and Carroll (2000) compared the in vivo function of the Ubx proteins from Drosophila melanogaster and the onychophoran species Acanthokara kaputensis. When the onychophoran $U b x$ is expressed during Drosophila development, it can activate and repress many of the same target genes as the Drosophila Ubx itself. However, two specific functions of the Drosophila Ubx, segmental identity of the embryonic ectoderm and the repression of Distalless-target genes, cannot be overtaken by the onychophoran Ubx protein (Grenier and Carroll 2000). Comparative work on arthropod and nematode Hox genes therefore indicates that despite the conservation of multiple protein functions, specific aspects of protein function do evolve. Like in the case of the nematode LIN-39 protein, functional divergence in the arthropod Ubx protein is also caused by sequence changes outside the homeodomain region (Grenier and Carroll 2000).

\section{Cel-lin-39, a direct target for MAPK phosphorylation?}

It has been shown previously that Cel-LIN-39 expression levels in the vulval cell lineage, especially in P6.p, are up-regulated following AC-induced activation of the MAPK signaling cascade (Maloof and Kenyon 1998). Given this position of Cel-LIN-39 downstream of MAPK signaling, it has been speculated that Cel-LIN-39 might also be a direct target for phosphorylation by MAPK (Eisenmann et al. 1998). The recently defined signal sequence for a MAPK docking site, FXFP, was identified in several C. elegans proteins as a prerequisite for efficient serine/threonine phosphorylation by MAPK (Jacobs et al. 1999). Our observation that the C-terminal domain of Cel-LIN-39 (but not Ppa-LIN-39) contains a close match to this signal sequence $\mathrm{C}$-terminal to a putative MAPK phosphorylation site is in agreement with the suggested role of Cel-LIN-39 as a direct MAPK target.

However, the present work indicates that mutation of neither the predicted phosphorylation site nor the signal sequence impaired the function of Cel-lin-39 in vulval development, suggesting that Cel-LIN-39 is not a target for MAPK. This was strengthened by demonstrating that a Cel-LIN-39 protein completely lacking the C-terminal domain had a rescuing activity identical to a full-length protein. When tested in vitro, no MAPK-dependent phosphorylation of Cel-LIN-39 could be observed, further corroborating the observation from the rescue experiments that Cel-LIN-39 might not be a target for MAPK phosphorylation. It is also worth noting that the sequence of the proteins experimentally investigated by Jacobs et al. (1999) only contained a subset of all possible amino acids at position 2 in the MAPK docking-site sequence FXFP. As the sequence FRFP, present in CelLIN-39, was not included in their study, it can be speculated that the docking-signal sequence is more restricted in position 2 than thought previously.

In summary, this study provides evidence that despite the limited conservation of protein sequences, changes of the developmental aspects of vulval formation do not rely on the molecular evolution of the Ppa-LIN-39 and Cel-LIN-39 proteins. However, it is too early to draw general conclusions about the relative importance of regulatory versus coding evolution. To obtain a better picture, more case studies are needed in which the function of homologous proteins is tested in in vivo assays in the context of phylogenetics and site-directed mutagenesis.

\section{Materials and methods}

\section{C. elegans strains and culture}

Strains were maintained under standard conditions as described (Brenner 1974). Wild-type C. elegans corresponds to Bristol, strain N2. The strain MT4498, with the genotype lin-39(n1880), was used for rescue experiments.

\section{Plasmid constructs}

All DNA constructs were made using standard techniques (Sambrook et al. 1989) and verified by restriction mapping or sequencing. In addition, all exons amplified by PCR were verified by sequencing. The basic 10-kb C. elegans lin-39 genomic construct pKG8 was generated by PCR amplification from the cosmid F44F12 and cloning of the PCR product into pBIIKS (Stratagene). This construct contains $4.2 \mathrm{~kb}$ upstream of the ATG and $73 \mathrm{bp}$ downstream of the poly(A) site. The 23-kb C. elegans genomic construct pKG9 was generated by extending pKG8 with an 8.7-kb PCR product in the $5^{\prime}$ end and a 3.9-kb PCR product in the $3^{\prime}$ end. The LacZ reporter gene was amplified from pPD90.31 (a gift from A. Fire, Carnegie Institute of Washington, Baltimore, MD) with primers introducing in-frame NcoI sites. This PCR fragment was subsequently cloned into pKG8 using the unique NcoI site located in exon 2 to create pKG1. The promoterless vector pKG2 was generated by digestion with AatII, which cuts 37 nt upstream of the transcription start site, and BamHI, followed by fill-in and religation. The vectors pKG4 and pKG5 were made by exchanging appropriate regions with corresponding fragments from the $C$. elegans lin-39 cDNA. pKG5 was made by cloning a C. elegans lin-39 promoter fragment into pPD95.07. This construct is a translational fusion retaining the start ATG from the lin-39 ORF. For creation of pKG6 the 3' UTR of the C. elegans lin-39 cDNA was amplified and cloned into the $3^{\prime}$ MCS of pPD95.11. 
To this vector the promoter region from pKG5 was added as a BamHI/DraIII fragment. To make pKG11 two PCR products were amplified from pKG8 with primers that introduce a SmaI site just $3^{\prime}$ of the ATG when the two PCR products are combined. These PCR products were cloned together into pBIIKS to generate a intermediary $10-\mathrm{kb}$ construct that subsequently was extended as for the generation of pKG9. A frame-shift mutation in exon 2 was generated by NcoI digestion, followed by fill-in and religation, leading to the final pKG11 vector. The Cel-lin39 and Ppa-lin-39 cDNAs were amplified by PCR, using 5' primers introducing SmaI sites replacing the ATG and 3' primers with SmaI sites downstream of the stop codons. These modified cDNAs were cloned into pKG11 to generate pKG12 and pKG13, respectively. The frame-shift mutation in pKG14 was introduced as for pKG11. Point mutations in pKG15-pKG19 were generated by the Quikchange technique (Stratagene) according to the manufacturer's instructions. Mutagenesis was performed on a small genomic subclone that, after sequencing, was reintroduced into the 23-kb background. The Ppa-lin-39 genomic construct pKG10 was derived from a previously isolated $\lambda$ clone (Eizinger and Sommer 1997) by transferring the entire insert to pBIIKS. This insert contains at least $10 \mathrm{~kb}$ of upstream sequence, all exons and introns, and approximately $300 \mathrm{bp}$ downstream of the poly(A) signal. The LacZ reporter in pKG7 was inserted as for pKG1. Detailed information about individual constructs is available upon request.

\section{Germ line transformation}

Germ line transformation and generation of transgenic C. elegans strains were performed as described (Mello et al. 1991; Mello and Fire 1995). The LacZ expression constructs were coinjected with rol-6(su1006) at a concentration of $50 \mathrm{ng} / \mu \mathrm{L}$ for each plasmid into wild-type C. elegans. Rescue constructs were injected into lin-39(n1880) with the coinjection marker pTG96 (Gu et al. 1998), encoding sur-5::GFP (a gift from M. Han, University of Colorado at Boulder, CO). The coinjection marker was kept at a concentration of $50 \mathrm{ng} / \mu \mathrm{L}$, whereas rescuing constructs were injected at 50 or $10 \mathrm{ng} / \mu \mathrm{L}$ with no observable difference in efficiency (data not shown). The total DNA concentration was kept constant by addition of pBIIKS. Some rescuing constructs were also tested at $1 \mathrm{ng} / \mu \mathrm{L}$ with the effect of fewer lines showing rescue. However, those lines with rescue had a similar degree of rescue as lines established with 10 or $50 \mathrm{ng} / \mathrm{\mu L}$ of the rescue construct (data not shown). For observations of VC neurons, pNE1, encoding unc-4::GFP (a gift from D. Miller), was used as marker. pNE1 is expressed in several neurons, including the VC neurons (Miller and Niemeyer 1995; Pflugrad et al. 1997; Lickteig et al. 2001). In these experiments pTG96 was replaced with $t$ tx-3::GFP (a gift from O. Hobert), which is only expressed in two neurons in the head (Hobert et al. 1997) and therefore does not interfere with VC neuron identification.

\section{LacZ-staining}

Staining of transgenic animals with LacZ reporter constructs was performed as described previously (Fire et al. 1990; Fire 1992). Animals were incubated in staining solution for $6 \mathrm{~h}$ to overnight at $37^{\circ} \mathrm{C}$.

\section{Egg-laying rescue assay}

Transgenic L2/L3 animals were picked singly to plates at day 1 and incubated at $25^{\circ} \mathrm{C}$. Eggs were counted daily until day 5 , where normally no or only a few eggs were still laid. Animals laying at least one egg were scored as rescued (non-Egl). Eggs and newly hatched larvae were counted and removed (alternatively, the mother was moved to a new plate) until the end of the experimental period or until the mother died from internal hatching of progeny. In most cases 20 animals per individual transgenic line were analyzed for 5 to 6 different lines per transgenic construct tested. Cel-lin-39(n1880) mutant animals are completely egg-laying defective and no eggs are laid.

\section{Lineage analysis}

Cell lineage was analyzed using Nomarski optics as described (Sulston and Horvitz 1977). Transgenic L2/L3 larvae were identified based on the expression of the GFP reporter. In isolated animals the presence or absence of P4-8.p and their fate $\left(1^{\circ}, 2^{\circ}\right.$, $3^{\circ}$, or fused) were scored.

\section{In vitro translation and phosphorylation}

Proteins were translated in vitro in the presence of unlabeled or ${ }^{35}$ S-labeled methionine using a TnT Quick Coupled Transcription/Translation system (Promega) according to the manufacturer's instructions. In vitro phosphorylation was performed as described previously (Jacobs et al. 1999) using recombinant $\mathrm{p} 42$ MAPK (Erk-2; New England Biolabs). Proteins labeled with ${ }^{35} \mathrm{~S}-$ methionine were phosphorylated with unlabeled ATP; unlabeled proteins were phosphorylated with $\left[\gamma^{-32} \mathrm{P}\right]$ ATP. Samples were separated on SDS-PAGE gels and subjected to autoradiography. The vector encoding human Elk-1, used as a positive control, was kindly provided by F.F. Wiebel.

\section{Acknowledgments}

We thank members of the laboratory for discussions and J. Srinivasan and F.F. Wiebel for helpful comments on the manuscript. The plasmids encoding unc-4::GFP, sur-5::GFP, and ttx3::GFP were kindly provided by D. Miller, M. Han, and O. Hobert, respectively. The strains used in this study were obtained from the Caenorhabditis Genetics Center, which is supported by the National Center for Research Resources of the National Institutes of Health. K.G. was supported by a postdoctoral fellowship from the Wenner-Gren Foundation.

The publication costs of this article were defrayed in part by payment of page charges. This article must therefore be hereby marked "advertisement" in accordance with 18 USC section 1734 solely to indicate this fact.

\section{References}

Anderson, P. and Kimble, J. 1997. mRNA and translation. In C. elegans II (ed. D.L. Riddle et al.), pp. 185-208. Cold Spring Harbor Laboratory Press, Cold Spring Harbor, NY.

Beitel, G.J., Tuck, S., Greenwald, I., and Horvitz, H.R. 1995. The Caenorhabditis elegans gene lin-1 encodes an ETS-domain protein and defines a branch of the vulval induction pathway. Genes \& Dev. 9: 3149-3162.

Brenner, S. 1974. The genetics of Caenorhabditis elegans. Genetics 77: 71-94.

The C. elegans Sequencing Consortium. 1998. Genome sequence of the nematode C. elegans: A platform for investigating biology. Science 282: 2012-2018.

Clandinin, T.R., Katz, W.S., and Sternberg, P.W. 1997. Caenorhabditis elegans hom-C genes regulate the response of vulval precursor cells to inductive signal. Dev. Biol. 182: $150-161$.

Clark, S.G., Chisholm, A.D., and Horvitz, H.R. 1993. Control of 
cell fates in the central body region of $C$. elegans by the homeobox gene lin-39. Cell 74: 43-55.

Eisenmann, D.M., Maloof, J.N., Simske, J.S., Kenyon, C., and Kim, S.K. 1998. The $\beta$-catenin homolog BAR-1 and LET-60 Ras coordinately regulate the Hox gene lin-39 during Caenorhabditis elegans vulval development. Development 125: 3667-3680.

Eizinger, A. and Sommer, R.J. 1997. The homeotic gene lin-39 and the evolution of nematode epidermal cell fates. Science 278: 452-455.

Eizinger, A., Jungblut, B., and Sommer, R.J. 1999. Evolutionary change in the functional specificity of genes. Trends Genet. 15: $197-202$.

Fire, A. 1992. Histochemical techniques for locating Escherichia coli $\beta$-galactosidase activity in transgenic organisms. Genet. Anal. Tech. Appl. 9: 151-158.

Fire, A., Harrison, S.W., and Dixon, D. 1990. A modular set of lacZ fusion vectors for studying gene expression in Caenorhabditis elegans. Gene 93: 189-198.

Golding, G.B. and Dean, A.M. 1998. The structural basis of molecular adaptation. Mol. Biol. Evol. 15: 355-369.

Greenwald, I. 1997. Development of the vulva. In C. elegans II (ed. D.L. Riddle et al.), pp. 519-542. Cold Spring Harbor Laboratory Press, Cold Spring Harbor, NY.

Grenier, J.K. and Carroll, S.B. 2000. Functional evolution of the Ultrabithorax protein. Proc. Nat1. Acad. Sci. 97: 704-709.

Gu, T., Orita, S., and Han, M. 1998. Caenorhabditis elegans sur-5, a novel but conserved protein, negatively regulates let-60 ras activity during vulval induction. Mol. Cell. Biol. 18: 4556-4564.

Hill, R.J. and Sternberg, P.W. 1992. The gene lin-3 encodes an inductive signal for vulval development in C. elegans. $\mathrm{Na}$ ture 358: 470-476.

Hobert, O., Mori, I., Yamashita, Y., Honda, H., Ohshima, Y., Liu, Y., and Ruvkun, G. 1997. Regulation of interneuron function in the C. elegans thermoregulatory pathway by the ttx-3 LIM homeobox gene. Neuron 19: 345-357.

Hunter, C.P. and Kenyon, C. 1996. Spatial and temporal controls target pal-1 blastomere-specification activity to a single blastomere lineage in C. elegans embryos. Cell 87: 217-226.

Jacobs, D., Glossip, D., Xing, H., Muslin, A.J., and Kornfeld, K. 1999. Multiple docking sites on substrate proteins form a modular system that mediates recognition by ERK MAP kinase. Genes \& Dev. 13: 163-175.

Janknecht, R., Ernst, W.H., Pingoud, V., and Nordheim, A. 1993. Activation of ternary complex factor Elk-1 by MAP kinases. EMBO I. 12: 5097-5104.

Kimble, J. 1981. Alterations in cell lineage following laser ablation of cells in the somatic gonad of Caenorhabditis elegans. Dev. Biol. 87: 286-300.

Kreitman, M. and Comeron, J.M. 1999. Coding sequence evolution. Curr. Opin. Genet. Dev. 9: 637-641.

Li, C. and Chalfie, M. 1990. Organogenesis in C. elegans: Positioning of neurons and muscles in the egg-laying system. Neuron 4: 681-695.

Lickteig, K.M., Duerr, J.S., Frisby, D.L., Hall, D.H., Rand, J.B., and Miller III, D.M. 2001. Regulation of neurotransmitter vesicles by the homeodomain protein UNC-4 and its transcriptional co-repressor UNC-37/Groucho in Caenorhabditis elegans cholinergic motor neurons. I. Neuroscience 21: 2001-2014.

Maloof, J.N. and Kenyon, C. 1998. The hox gene lin-39 is required during $C$. elegans vulval induction to select the outcome of ras signaling. Development 125: 181-190.

Mello, C.C. and Fire, A. 1995. DNA transformation. In Caenorhabditis elegans: Modern biological analysis of an organ- ism (ed. H.F. Epstein and D.C. Shakes), pp. 341-348. Academic Press, New York.

Mello, C.C., Kramer, J.M., Stinchcomb, D., and Ambros, V. 1991. Efficient gene transfer in C. elegans: Extrachromosomal maintenance and integration of transforming sequences. EMBO J. 10: 3959-3970.

Miller III, D.M. and Niemeyer, C.J. 1995. Expression of the unc-4 homeoprotein in Caenorhabditis elegans motor neurons specifies presynaptic input. Development 121: 28772886.

Pflugrad, A., Meir, J.Y., Barnes, T.M., and Miller III, D.M. 1997. The Groucho-like transcription factor UNC-37 functions with the neural specificity gene unc-4 to govern motor neuron identity in C. elegans. Development 124: 1699-1709.

Pulak, R. and Anderson, P. 1993. mRNA surveillance by the Caenorhabditis elegans smg genes. Genes \& Dev. 7: 18851897.

Salser, S.J., Loer, C.M., and Kenyon, C. 1993. Multiple HOM-C gene interactions specify cell fates in the nematode central nervous system. Genes \& Dev. 7: 1714-1724.

Sambrook, J., Fritsch, E.F., and Maniatis, T. 1989. Molecular Cloning. A laborarory manual. Cold Spring Harbor Laboratory press, Cold Spring Harbor, NY.

Sommer, R.J. 2000. Evolution of nematode development. Curr. Opin. Genet. Dev. 10: 443-448.

Sommer, R.J. and Sternberg, P.W. 1996. Apoptosis and change of competence limit the size of the vulva equivalence group in Pristionchus pacificus-A genetic analysis. Curr. Biol. 6: 52-59.

Sommer, R.J., Carta, L., Kim, S.-Y., and Sternberg, P.W. 1996. Morphological, genetic and molecular description of Pristionchus pacificus sp. n. (Nematoda: Neodiplogastridae). Fundam. Appl. Nematol. 19: 511-521.

Sommer, R.J., Eizinger, A., Lee, K.Z., Jungblut, B., Bubeck, A., and Schlak, I. 1998. The Pristionchus HOX gene Ppa-lin-39 inhibits programmed cell death to specify the vulva equivalence group and is not required during vulval induction. Development 125: 3865-3873.

Sternberg, P.W. and Horvitz, H.R. 1986. Pattern formation during vulval development in C. elegans. Cell 44: 761-772.

Sulston, J.E. and Horvitz, H.R. 1977. Post-embryonic cell lineages of the nematode, Caenorhabditis elegans. Dev. Biol. 56: $110-156$.

Tan, P.B., Lackner, M.R., and Kim, S.K. 1998. MAP kinase signaling specificity mediated by the LIN-1 Ets/LIN-31 WH transcription factor complex during C. elegans vulval induction. Cell 93: 569-580.

Tautz, D. 2000. Evolution of transcriptional regulation. Curr. Opin. Genet. Dev. 10: 575-579.

Wang, B.B., Muller-Immergluck, M.M., Austin, J., Robinson, N.T., Chisholm, A., and Kenyon, C. 1993. A homeotic gene cluster patterns the anteroposterior body axis of C. elegans. Cell 74: 29-42.

Yang, S.H., Yates, P.R., Whitmarsh, A.J., Davis, R.J., and Sharrocks, A.D. 1998. The Elk-1 ETS-domain transcription factor contains a mitogen-activated protein kinase targeting motif. Mol. Cell. Biol. 18: 710-720. 


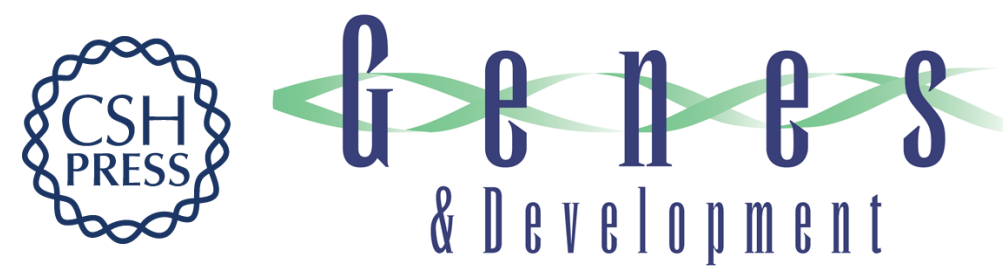

\section{Functional comparison of the nematode Hox gene lin-39 in C. elegans and $P$. pacificus reveals evolutionary conservation of protein function despite divergence of primary sequences}

Kaj Grandien and Ralf J. Sommer

Genes Dev. 2001, 15:

Access the most recent version at doi:10.1101/gad.200601

\section{References This article cites 38 articles, 16 of which can be accessed free at: http://genesdev.cshlp.org/content/15/16/2161.full.html\#ref-list-1}

License Email Alerting
Service $\begin{aligned} & \text { Receive free email alerts when new articles cite this article - sign up in the box at the top } \\ & \text { right corner of the article or click here. }\end{aligned}$

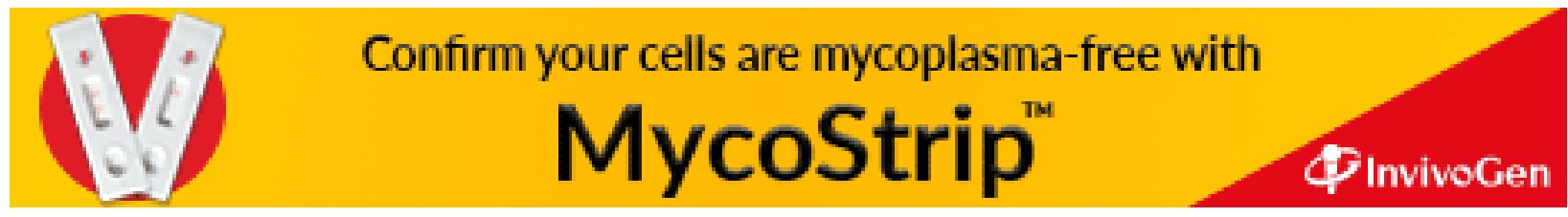

